\title{
ON THE QUANTITATIVE METRIC THEORY OF CONTINUED FRACTIONS IN POSITIVE CHARACTERISTIC
}

\author{
POJ LERTCHOOSAKUL ${ }^{1}$ AND RADHAKRISHNAN NAIR ${ }^{2 *}$ \\ ${ }^{1}$ Instytut Matematyki, Uniwersytet Gdanski, ul. Wita Stwosza 57, 80-308 Gdansk, \\ Poland (lertchoosakul@mat.ug.edu.pl) \\ ${ }^{2}$ Mathematical Sciences, the University of Liverpool, Peach Street, \\ Liverpool L69 7ZL, UK (nair@liv.ac.uk)
}

(Received 16 August 2016)

Abstract Let $\mathbb{F}_{q}$ be the finite field of $q$ elements. An analogue of the regular continued fraction expansion for an element $\alpha$ in the field of formal Laurent series over $\mathbb{F}_{q}$ is given uniquely by

$$
\alpha=A_{0}(\alpha)+\frac{1}{A_{1}(\alpha)+\frac{1}{A_{2}(\alpha)+\ddots}},
$$

where $\left(A_{n}(\alpha)\right)_{n=0}^{\infty}$ is a sequence of polynomials with coefficients in $\mathbb{F}_{q}$ such that $\operatorname{deg}\left(A_{n}(\alpha)\right) \geqslant 1$ for all $n \geqslant 1$. In this paper, we provide quantitative versions of metrical results regarding averages of partial quotients. A sample result we prove is that, given any $\epsilon>0$, we have

$$
\left|A_{1}(\alpha) \cdots A_{N}(\alpha)\right|^{1 / N}=q^{q /(q-1)}+o\left(N^{-1 / 2}(\log N)^{3 / 2+\epsilon}\right)
$$

for almost everywhere $\alpha$ with respect to Haar measure.

Keywords: continued fractions; metric theory of numbers

2010 Mathematics subject classification: Primary 11K50

Secondary 28D99

\section{Introduction}

Let $\mathbb{F}_{q}$ denote the finite field of $q$ elements, where $q$ is a power of a prime $p$. If $Z$ is an indeterminate, we denote by $\mathbb{F}_{q}[Z]$ and $\mathbb{F}_{q}(Z)$ the ring of polynomials in $Z$ with coefficients in $\mathbb{F}_{q}$ and the quotient field of $\mathbb{F}_{q}[Z]$, respectively. For each $P, Q \in \mathbb{F}_{q}[Z]$ with $Q \neq 0$, define $|P / Q|=q^{\operatorname{deg}(P)-\operatorname{deg}(Q)}$ and $|0|=0$. The field $\mathbb{F}_{q}\left(\left(Z^{-1}\right)\right)$ of formal Laurent series is the completion of $\mathbb{F}_{q}(Z)$ with respect to the valuation $|\cdot|$. That is,

$$
\mathbb{F}_{q}\left(\left(Z^{-1}\right)\right)=\left\{a_{n} Z^{n}+a_{n-1} Z^{n-1}+\cdots+a_{0}+a_{-1} Z^{-1}+\cdots: n \in \mathbb{Z}, a_{i} \in \mathbb{F}_{q}\right\}
$$

* Corresponding author.

(C) 2018 The Edinburgh Mathematical Society. This is an Open Access article, distributed under the terms of the Creative Commons Attribution licence (http://creativecommons.org/licenses/ by/4.0/), which permits unrestricted re-use, distribution, and reproduction in any medium, provided the original work is properly cited. 
and we have $\left|a_{n} Z^{n}+a_{n-1} Z^{n-1}+\cdots\right|=q^{n}\left(a_{n} \neq 0\right)$ and $|0|=0$, where $q$ is defined on the previous page. It is worth keeping in mind that $|\cdot|$ is a non-Archimedean norm, since $|\alpha+\beta| \leqslant \max (|\alpha|,|\beta|)$. In fact, $\mathbb{F}_{q}\left(\left(Z^{-1}\right)\right)$ is a non-Archimedean local field of positive characteristic $p$. As a result, up to a positive multiplicative constant, there exists a unique countably additive Haar measure $\mu$ on the Borel subsets of $\mathbb{F}_{q}\left(\left(Z^{-1}\right)\right)$. In $[\mathbf{1 0}$, pp. 65-70], Sprindžuk gives a characterization of Haar measure on $\mathbb{F}_{q}\left(\left(Z^{-1}\right)\right)$ by its value on the balls $B\left(\alpha ; q^{n}\right)=\left\{\beta \in \mathbb{F}_{q}\left(\left(Z^{-1}\right)\right):|\alpha-\beta|<q^{n}\right\}$. Indeed, it is shown that the equation $\mu\left(B\left(\alpha ; q^{n}\right)\right)=q^{n}$ completely characterizes Haar measure.

As in the classical context of real numbers, we have a continued fraction algorithm in $\mathbb{F}_{q}\left(\left(Z^{-1}\right)\right)$. Note that, in the case of the field of formal Laurent series, the roles of $\mathbb{Z}$, $\mathbb{Q}$ and $\mathbb{R}$ in the classical theory of continued fractions are played by $\mathbb{F}_{q}[Z], \mathbb{F}_{q}(Z)$ and $\mathbb{F}_{q}\left(\left(Z^{-1}\right)\right)$, respectively. For each $\alpha \in \mathbb{F}_{q}\left(\left(Z^{-1}\right)\right)$, we can write

$$
\alpha=A_{0}+\frac{1}{A_{1}+\frac{1}{A_{2}+\ddots}}=\left[A_{0} ; A_{1}, A_{2}, \ldots\right],
$$

where $\left(A_{n}\right)_{n=0}^{\infty}$ is a sequence of polynomials in $\mathbb{F}_{q}[Z]$ with $\left|A_{n}\right|>1$ for all $n \geqslant 1$. Here the sequence $\left(A_{n}\right)_{n \geq 0}$ is uniquely determined by $\alpha$ for $\alpha$ not in $\mathbb{F}_{q}(Z)$. Note that, in the context of continued fractions, we shall often deal with the set $\mathbb{F}_{q}[Z]^{*}=\left\{A \in \mathbb{F}_{q}[Z]\right.$ : $|A|>1\}$. As in the classical theory, we define recursively the two sequences of polynomials $\left(P_{n}\right)_{n=0}^{\infty}$ and $\left(Q_{n}\right)_{n=0}^{\infty}$ by

$$
P_{n}=A_{n} P_{n-1}+P_{n-2} \text { and } Q_{n}=A_{n} Q_{n-1}+Q_{n-2},
$$

with the initial conditions $P_{0}=A_{0}, Q_{0}=1, P_{1}=A_{1} A_{0}+1$ and $Q_{1}=A_{1}$. Then we have $Q_{n} P_{n-1}-P_{n} Q_{n-1}=(-1)^{n}$, and whence $P_{n}$ and $Q_{n}$ are coprime. In addition, we have $P_{n} / Q_{n}=\left[A_{0} ; A_{1}, \ldots, A_{n}\right]$. For a general reference on this subject, the reader should consult $[\mathbf{6}, \mathbf{9}]$.

The Gauss map, or the continued fraction map, $T$ on the unit ball $B(0 ; 1)=\left\{a_{-1} Z^{-1}+\right.$ $\left.a_{-2} Z^{-2}+\cdots: a_{i} \in \mathbb{F}_{q}\right\}$ is defined by

$$
T \alpha=\left\{\frac{1}{\alpha}\right\} \quad \text { and } \quad T 0=0,
$$

where $\left\{a_{n} Z^{n}+\cdots+a_{0}+a_{-1} Z^{-1}+\cdots\right\}=a_{-1} Z^{-1}+a_{-2} Z^{-2}+\cdots$ denotes its fractional part. We note that if $\alpha=\left[0 ; A_{1}(\alpha), A_{2}(\alpha), \ldots\right]$, then we have,

$$
T^{n} \alpha=\left[0 ; A_{n+1}(\alpha), A_{n+2}(\alpha), \ldots\right] \quad \text { and } \quad A_{m}\left(T^{n} \alpha\right)=A_{n+m}(\alpha)
$$

for all $m \geqslant 1$ and $n \geqslant 0$. It was proved in [7] that the map $T$ is measure-preserving and exact with respect to Haar measure $\mu$. This fact of exactness implies all order of mixing properties and in particular ergodicity. It was shown by Niederreiter [8] that $T$ in fact has a natural extension that is Bernoulli. Of course this implies the exactness of $T$. See $[\mathbf{7}, \mathbf{8}]$ respectively for the statement of the exactness and Bernoulli properties.

In this setting, Houndonougbo [4] and Berthé and Nakada [1] were able to establish, by using Birkhoff's ergodic theorem, some qualitative metrical results on the averages of 
partial quotients of continued fraction expansions. For instance, they proved the positive characteristic analogue of Khinchin's famous result that

$$
\lim _{N \rightarrow \infty}\left|A_{1}(\alpha) \cdots A_{N}(\alpha)\right|^{1 / N}=q^{q /(q-1)}
$$

for almost everywhere $\alpha \in B(0 ; 1)$ with respect to Haar measure.

In this paper, we investigate quantitative versions of metrical results regarding ergodic averages. In particular we find the error term in (1.1) as a function of $N$. In other words, we shall see how the geometric means of $\left|A_{n}(\alpha)\right|(n=1, \ldots, N)$ deviates from the positive characteristic Khinchin's constant $q^{q /(q-1)}$ for almost everywhere $\alpha$. This is an extension of work of de Vroedt [2] to the field of formal Laurent series.

To deal with the error terms, we need some notation to describe asymptoticity. Given two real functions $f_{1}$ and $f_{2}$ and a positive function $g$ defined on $\mathbb{N}$, we write $f_{1}=$ $f_{2}+O(g)$ if $\left|f_{1}-f_{2}\right|<c g$ for some positive constant $c$, and we write $f_{1}=f_{2}+o(g)$ if $\lim _{N \rightarrow \infty}\left(f_{1}(N)-f_{2}(N)\right) / g(N)=0$.

We now summarize the contents of this paper. In $\S 2$, we state several quantitative metrical results on the behaviour of averages of partial quotients of continued fraction expansions in positive characteristic. In $\S 3$, we describe Gál and Koksma's method for determining the error term of ergodic averages, and we give some lemmas necessary for proving the quantitative metrical theorems of continued fractions. In $\S 4$, we give the proofs of all the statements that appear in $\S 2$.

\section{Quantitative metrical theorems}

The proofs of the following statements will be given in $\S 4$. We start with the first two general theorems for calculating the quantitative ergodic averages.

Theorem 1. Suppose that $F: \mathbb{R}_{\geqslant 0} \rightarrow \mathbb{R}$ is a function such that

$$
\int_{B(0 ; 1)}\left|F\left(\left|A_{1}(x)\right|\right)\right|^{2} \mathrm{~d} \mu(x)<\infty
$$

Then, given any $\epsilon>0$, we have

$$
\frac{1}{N} \sum_{n=1}^{N} F\left(\left|A_{1}\left(T^{n} \alpha\right)\right|\right)=\int_{B(0 ; 1)} F\left(\left|A_{1}(x)\right|\right) \mathrm{d} \mu(x)+o\left(N^{-1 / 2}(\log N)^{3 / 2+\epsilon}\right)
$$

for almost everywhere $\alpha \in B(0 ; 1)$ with respect to Haar measure.

Theorem 2. Suppose that $H: \mathbb{N}^{m} \rightarrow \mathbb{R}$ is a function such that

$$
\int_{B(0 ; 1)}\left|H\left(\left|A_{1}(x)\right|,\left|A_{2}(x)\right|, \ldots,\left|A_{m}(x)\right|\right)\right|^{2} \mathrm{~d} \mu(x)<\infty .
$$


Then, given any $\epsilon>0$, we have

$$
\begin{aligned}
& \frac{1}{N} \sum_{n=1}^{N} H\left(\left|A_{1}\left(T^{n} \alpha\right)\right|,\left|A_{2}\left(T^{n} \alpha\right)\right|, \ldots,\left|A_{m}\left(T^{n} \alpha\right)\right|\right) \\
& \quad=\sum_{\left(i_{1}, \ldots, i_{m}\right) \in \mathbb{N}^{m}} H\left(q^{i_{1}}, \ldots, q^{i_{m}}\right)\left(\frac{(q-1)^{m}}{q^{i_{1}+\cdots+i_{m}}}\right)+o\left(N^{-1 / 2}(\log N)^{3 / 2+\epsilon}\right)
\end{aligned}
$$

for almost everywhere $\alpha \in B(0 ; 1)$ with respect to Haar measure.

Theorems 1 and 2 are general results for calculating means. Specializing for instance to the case $F(x)=\log _{q} x$, we establish the quantitative version of the positive characteristic Khinchin's constant

$$
\left|A_{1}(\alpha) \cdots A_{N}(\alpha)\right|^{1 / N}=q^{q /(q-1)}+o\left(N^{-1 / 2}(\log N)^{3 / 2+\epsilon}\right)
$$

for almost everywhere $\alpha \in B(0 ; 1)$ with respect to Haar measure, $[\mathbf{2}, \mathbf{6}]$. Results for means other than the geometric mean can be obtained by making different choices of $F$ and $H$, see [5, pp. 230-232] for more details.

The following three theorems can be viewed as corollaries of Theorem 1 . We note that they should be compared with Theorems 12-14 of [7] as they sharpen those results when $\left(a_{j}\right)_{j=1}^{\infty}$ is considered as the sequence of natural numbers in the literature.

Theorem 3. Given any $\epsilon>0$, we have

$$
\frac{1}{N} \sum_{n=1}^{N} \operatorname{deg}\left(A_{n}(\alpha)\right)=\frac{q}{q-1}+o\left(N^{-1 / 2}(\log N)^{3 / 2+\epsilon}\right)
$$

for almost everywhere $\alpha \in B(0 ; 1)$ with respect to Haar measure.

Theorem 4. Given any $A \in \mathbb{F}_{q}[Z]^{*}$ and $\epsilon>0$, we have

$$
\frac{1}{N} \cdot \#\left\{1 \leqslant n \leqslant N: A_{n}(\alpha)=A\right\}=|A|^{-2}+o\left(N^{-1 / 2}(\log N)^{3 / 2+\epsilon}\right)
$$

for almost everywhere $\alpha \in B(0 ; 1)$ with respect to Haar measure.

Theorem 5. Let $k<l$ be two natural numbers. Given any $\epsilon>0$, we have

$$
\begin{aligned}
& \frac{1}{N} \cdot \#\left\{1 \leqslant n \leqslant N: \operatorname{deg}\left(A_{n}(\alpha)\right)=l\right\}=\frac{q-1}{q^{l}}+o\left(N^{-1 / 2}(\log N)^{3 / 2+\epsilon}\right), \\
& \frac{1}{N} \cdot \#\left\{1 \leqslant n \leqslant N: \operatorname{deg}\left(A_{n}(\alpha)\right) \geqslant l\right\}=\frac{1}{q^{l-1}}+o\left(N^{-1 / 2}(\log N)^{3 / 2+\epsilon}\right), \\
& \frac{1}{N} \cdot \#\left\{1 \leqslant n \leqslant N: k \leqslant \operatorname{deg}\left(A_{n}(\alpha)\right)<l\right\}=\frac{1}{q^{k-1}}\left(1-\frac{1}{q^{l-k}}\right)+o\left(N^{-1 / 2}(\log N)^{3 / 2+\epsilon}\right)
\end{aligned}
$$

for almost everywhere $\alpha \in B(0 ; 1)$ with respect to Haar measure. 


\section{Lemmas}

In this section, we collect some results that are necessary for establishing the quantitative metric theory of continued fractions in positive characteristic.

To begin with, we introduce Gál and Koksma's method for determining the error term of ergodic averages. The following lemma appears in $[\mathbf{3}]$ in slightly different language.

Lemma 6 ([3, Théorème 3$])$. Let $S$ be a measurable set. For any non-negative integers $M$ and $N$, let $\varphi(M, N ; x) \geqslant 0$ be a function defined on $S$ such that

(i) $\varphi(M, 0 ; x)=0$ for all $M \geqslant 0$;

(ii) $\varphi(M, N ; x) \leqslant \varphi\left(M, N^{\prime} ; x\right)+\varphi\left(M+N^{\prime}, N-N^{\prime} ; x\right)$ for all $M, N \geqslant 0$ and $0 \leqslant$ $N^{\prime} \leqslant N$.

Suppose that, for all $M \geqslant 0$,

$$
\int_{S} \varphi(M, N ; x)^{p} \mathrm{~d} x=O(\phi(N)),
$$

where $\phi(N) / N$ is a non-decreasing function. Then, given any $\epsilon>0$, we have

$$
\varphi(0, N ; x)=o\left(\phi(N)^{1 / p}(\log N)^{1+1 / p+\epsilon}\right)
$$

for almost everywhere $x \in S$.

Before proceeding, we give the following two remarks on Lemma 6. First, Gál and Koksma stated their results in the setting where the set $S$ is a measurable subset of a Euclidean space. None of the proofs however in [3] depend on the Euclidean setting. In fact, their result is true more generally. We are interested in the case where $S=B(0 ; 1)$, for which the result is also true. Second, the function $\varphi$ can be viewed as a generalization of the difference of two functions in a sequence:

$$
\varphi(M, N ; x)=\left|\varphi_{M+N}(x)-\varphi_{M}(x)\right|,
$$

where property (ii) is just a generalization of the triangle inequality

$$
\left|\varphi_{M+N}(x)-\varphi_{M}(x)\right| \leqslant\left|\varphi_{M+N^{\prime}}(x)-\varphi_{M}(x)\right|+\left|\varphi_{M+N}(x)-\varphi_{M+N^{\prime}}(x)\right| .
$$

Particularly, we focus on the case where $\varphi_{N}(x)=\sum_{n=1}^{N} F_{n}(x)$ and where $\left(F_{n}\right)_{n=1}^{\infty}$ is a sequence of functions defined on $S$; that is,

$$
\varphi(M, N ; x)=\sum_{n=M+1}^{M+N} F_{n}(x) .
$$

Next, we introduce the notion of a cylinder in positive characteristic and its fundamental properties. Recall that $\mathbb{F}_{q}[Z]^{*}=\left\{A \in \mathbb{F}_{q}[Z]:|A|>1\right\}$. Let $n$ be a natural number, and let $A_{1}, \ldots, A_{n} \in \mathbb{F}_{q}[Z]^{*}$. The cylinder $\Delta_{A_{1}, \ldots, A_{n}}$ of length $n$ is defined to be the set of all 
points in $B(0 ; 1)$ whose continued fraction expansions are of the form $\left[0 ; A_{1}, \ldots, A_{n}, \ldots\right]$. That is,

$$
\Delta_{A_{1}, \ldots, A_{n}}=\left\{\left[0 ; A_{1}, \ldots, A_{n-1}, A_{n}+\beta\right]: \beta \in B(0 ; 1)\right\} .
$$

We now show how a cylinder can be seen as a ball. This is crucial for calculating the measure of each cylinder.

Lemma 7 ([7, Lemma 2]). For all $n \in \mathbb{N}$ and $A_{1}, \ldots, A_{n} \in \mathbb{F}_{q}[Z]^{*}$, we have

$$
\Delta_{A_{1}, \ldots, A_{n}}=B\left(\left[0 ; A_{1}, \ldots, A_{n}\right] ;\left|A_{1} \cdots A_{n}\right|^{-2}\right) .
$$

From Lemma 7 , it follows immediately that $\mu\left(\Delta_{A_{1}, \ldots, A_{n}}\right)=\left|A_{1} \cdots A_{n}\right|^{-2}$. We note also that two cylinders $\Delta_{A_{1}, \ldots, A_{n}}$ and $\Delta_{B_{1}, \ldots, B_{n}}$ are disjoint if and only if $A_{j} \neq B_{j}$ for some $1 \leqslant j \leqslant n$.

The notion of a cylinder is an effective tool because of the following fact. Let $\mathcal{A}$ denote the algebra of finite unions of cylinders. Then $\mathcal{A}$ generates the Borel $\sigma$-algebra of the dynamical system $(B(0 ; 1), \mathcal{B}, \mu, T)$. This follows from the fact that the cylinders are clearly Borel sets themselves and that they separate points, that is, if $\alpha \neq \beta$, then there exist disjoint cylinders $\Delta_{1}$ and $\Delta_{2}$ such that $\alpha \in \Delta_{1}$ and $\beta \in \Delta_{2}$.

Our final lemma will be useful when we would like to change variables in an integration. This result follows immediately from the fact that the map $T$ is measure-preserving, $[\mathbf{7}$, Lemma 3].

Lemma 8. For all $n \in \mathbb{N}$, we have $\mathrm{d} \mu\left(T^{-n} x\right)=\mathrm{d} \mu(x)$.

\section{Proofs}

Proof of Theorem 1. Consider Lemma 6 with $S=B(0 ; 1)$,

$$
\varphi(M, N ; \alpha)=\left|\sum_{n=M+1}^{M+N}\left(F\left(\left|A_{1}\left(T^{n} \alpha\right)\right|\right)-\int_{B(0 ; 1)} F\left(\left|A_{1}(x)\right|\right) \mathrm{d} \mu(x)\right)\right|,
$$

$\phi(N)=N$ and $p=2$. First we check that this function $\varphi$ satisfies the hypotheses (i) and (ii) of Lemma 6 . It is clear by the notation of summation that, for all $M \geqslant 0$, we have

$$
\varphi(M, 0 ; \alpha)=\left|\sum_{n=M+1}^{M}\left(F\left(\left|A_{1}\left(T^{n} \alpha\right)\right|\right)-\int_{B(0 ; 1)} F\left(\left|A_{1}(x)\right|\right) \mathrm{d} \mu(x)\right)\right|=0 .
$$

Moreover, by the triangle inequality, we have

$$
\begin{aligned}
\varphi(M, N ; \alpha) & =\left|\sum_{n=M+1}^{M+N}\left(F\left(\left|A_{1}\left(T^{n} \alpha\right)\right|\right)-\int_{B(0 ; 1)} F\left(\left|A_{1}(x)\right|\right) \mathrm{d} \mu(x)\right)\right| \\
& \leqslant\left|\sum_{n=M+1}^{M+N^{\prime}}\left(F\left(\left|A_{1}\left(T^{n} \alpha\right)\right|\right)-\int_{B(0 ; 1)} F\left(\left|A_{1}(x)\right|\right) \mathrm{d} \mu(x)\right)\right|
\end{aligned}
$$




$$
\begin{aligned}
& +\left|\sum_{n=M+N^{\prime}+1}^{M+N}\left(F\left(\left|A_{1}\left(T^{n} \alpha\right)\right|\right)-\int_{B(0 ; 1)} F\left(\left|A_{1}(x)\right|\right) \mathrm{d} \mu(x)\right)\right| \\
& =\varphi\left(M, N^{\prime} ; \alpha\right)+\varphi\left(M+N^{\prime}, N-N^{\prime} ; \alpha\right)
\end{aligned}
$$

for all $M, N \geqslant 0$ and $0 \leqslant N^{\prime} \leqslant N$.

Now the proof is reduced to showing that, for any pair of integers $M \geqslant 0$ and $N \geqslant 1$, we have

$$
I=\int_{B(0 ; 1)}\left|\sum_{n=M+1}^{M+N}\left(F\left(\left|A_{1}\left(T^{n} \alpha\right)\right|\right)-\int_{B(0 ; 1)} F\left(\left|A_{1}(x)\right|\right) \mathrm{d} \mu(x)\right)\right|^{2} \mathrm{~d} \mu(\alpha) \leqslant K N,
$$

where $K$ is a constant depending only on $F(x)$.

Put

$$
P_{1}=\int_{B(0 ; 1)} F\left(\left|A_{1}(x)\right|\right) \mathrm{d} \mu(x) \quad \text { and } \quad P_{2}=\int_{B(0 ; 1)} F\left(\left|A_{1}(x)\right|\right)^{2} \mathrm{~d} \mu(x) .
$$

To calculate $P_{1}$ and $P_{2}$, we note that $B(0 ; 1)$ can be partitioned into a disjoint union of cylinders of length one. Indeed, we have

$$
B(0 ; 1)=\bigcup_{n=1}^{\infty} \bigcup_{\substack{A \in \mathbb{F}_{q}[Z]^{*} \\|A|=q^{n}}} \Delta_{A}
$$

We also know that there are distinct $(q-1) q^{n}$ cylinders $\Delta_{A}$ with $|A|=q^{n}$ and whose measures are $\mu\left(\Delta_{A}\right)=q^{-2 n}$. It now follows that

$$
P_{1}=\sum_{n=1}^{\infty} \sum_{\substack{A \in \mathbb{F}_{q}[Z]^{*} \\|A|=q^{n}}} \int_{\Delta_{A}} F\left(\left|A_{1}(x)\right|\right) \mathrm{d} \mu(x)=\sum_{n=1}^{\infty} \frac{q-1}{q^{n}} F\left(q^{n}\right)=(q-1) \sum_{n=1}^{\infty} \frac{F\left(q^{n}\right)}{q^{n}}
$$

and

$$
P_{2}=\sum_{n=1}^{\infty} \sum_{\substack{A \in \mathbb{F}_{q}[Z]^{*} \\|A|=q^{n}}} \int_{\Delta_{A}} F\left(\left|A_{1}(x)\right|\right)^{2} \mathrm{~d} \mu(x)=(q-1) \sum_{n=1}^{\infty} \frac{F\left(q^{n}\right)^{2}}{q^{n}}
$$

Working out $I=\int_{B(0 ; 1)}\left(\sum_{n=M+1}^{M+N}\left(F\left(\left|A_{1}\left(T^{n} \alpha\right)\right|\right)-P_{1}\right)\right)^{2} \mathrm{~d} \mu(\alpha)$, we get

$$
\begin{aligned}
I= & \sum_{n=M+1}^{M+N} \int_{B(0 ; 1)}\left(F\left(\left|A_{1}\left(T^{n} \alpha\right)\right|\right)-P_{1}\right)^{2} \mathrm{~d} \mu(\alpha) \\
& +2 \sum_{n=M+1}^{M+N-1} \sum_{\substack{m=M+2 \\
m>n}}^{M+N} \int_{B(0 ; 1)}\left(F\left(\left|A_{1}\left(T^{n} \alpha\right)\right|\right)-P_{1}\right)\left(F\left(\left|A_{1}\left(T^{m} \alpha\right)\right|\right)-P_{1}\right) \mathrm{d} \mu(\alpha) .
\end{aligned}
$$


By Lemma 8, we can use the change of variables formula to obtain

$$
\begin{aligned}
& \sum_{n=M+1}^{M+N} \int_{B(0 ; 1)}\left(F\left(\left|A_{1}\left(T^{n} \alpha\right)\right|\right)-P_{1}\right)^{2} \mathrm{~d} \mu(\alpha) \\
& =\sum_{n=M+1}^{M+N}\left(\int_{B(0 ; 1)} F\left(\left|A_{1}\left(T^{n} \alpha\right)\right|\right)^{2} \mathrm{~d} \mu(\alpha)-2 P_{1} \int_{B(0 ; 1)} F\left(\left|A_{1}\left(T^{n} \alpha\right)\right|\right) \mathrm{d} \mu(\alpha)+P_{1}^{2}\right) \\
& =\sum_{n=M+1}^{M+N}\left(\int_{B(0 ; 1)} F\left(\left|A_{1}(\alpha)\right|\right)^{2} \mathrm{~d} \mu\left(T^{-n} \alpha\right)\right. \\
& =\sum_{n=M+1}^{M+N}\left(\int_{B(0 ; 1)} F\left(\left|A_{1}(\alpha)\right|\right)^{2} \mathrm{~d} \mu(\alpha)-2 P_{1} \int_{B(0 ; 1)} F\left(\left|A_{1}(\alpha)\right|\right) \mathrm{d} \mu(\alpha)+P_{1}^{2}\right) \\
& =\sum_{n=M+1}^{M+N}\left(P_{2}-2 P_{1}^{2}+P_{1}^{2}\right)=N\left(P_{2}-P_{1}^{2}\right)
\end{aligned}
$$

and

$$
\begin{aligned}
& \sum_{n=M+1}^{M+N-1} \sum_{\substack{m=M+2 \\
m>n}}^{M+N} \int_{B(0 ; 1)}\left(F\left(\left|A_{1}\left(T^{n} \alpha\right)\right|\right)-P_{1}\right)\left(F\left(\left|A_{1}\left(T^{m} \alpha\right)\right|\right)-P_{1}\right) \mathrm{d} \mu(\alpha) \\
& =\sum_{n=M+1}^{M+N-1} \sum_{\substack{m=M+2 \\
m>n}}^{M+N}\left(\int_{B(0 ; 1)} F\left(\left|A_{1}\left(T^{n} \alpha\right)\right|\right) F\left(\left|A_{1}\left(T^{m} \alpha\right)\right|\right) \mathrm{d} \mu(\alpha)\right. \\
& -P_{1} \int_{B(0 ; 1)} F\left(\left|A_{1}\left(T^{n} \alpha\right)\right|\right) \mathrm{d} \mu(\alpha) \\
& =\sum_{n=M+1}^{M+N-1} \sum_{\substack{m=M+N \\
m>n}}^{M+P_{1}}\left(\int_{B(0 ; 1)} F\left(\left|A_{1}\left(T^{m} \alpha\right)\right|\right) \mathrm{d} \mu(\alpha)+P_{1}^{2}\right) \\
& =\sum_{n=1}^{N-1}(N-n)\left(\int_{B(0 ; 1)} F\left(\left|A_{1}(\alpha)\right|\right) F\left(\left|A_{1}\left(T^{n} \alpha\right)\right|\right) \mathrm{d} \mu(\alpha)-P_{1}^{2}\right) .
\end{aligned}
$$

Combining (4.4)-(4.6), we now have

$$
I=N\left(P_{2}-P_{1}^{2}\right)+2 \sum_{n=1}^{N-1}(N-n)\left(\int_{B(0 ; 1)} F\left(\left|A_{1}(\alpha)\right|\right) F\left(\left|A_{1}\left(T^{n} \alpha\right)\right|\right) \mathrm{d} \mu(\alpha)-P_{1}^{2}\right) .
$$


We can calculate $\int_{B(0 ; 1)} F\left(\left|A_{1}(\alpha)\right|\right) F\left(\left|A_{1}\left(T^{n} \alpha\right)\right|\right) \mathrm{d} \mu(\alpha)$ explicitly as follows

$$
\begin{aligned}
& \int_{B(0 ; 1)} F\left(\left|A_{1}(\alpha)\right|\right) F\left(\left|A_{1}\left(T^{n} \alpha\right)\right|\right) \mathrm{d} \mu(\alpha) \\
& =\sum_{A_{2}, \ldots, A_{n} \in \mathbb{F}_{q}[Z]^{*}}\left|A_{2} \cdots A_{n}\right|^{-2}\left(\sum_{i=1}^{\infty}\left(\frac{q-1}{q^{i}}\right) F\left(q^{i}\right)\right)\left(\sum_{j=1}^{\infty}\left(\frac{q-1}{q^{j}}\right) F\left(q^{j}\right)\right) \\
& =(q-1)^{2}\left(\sum_{i=1}^{\infty} \frac{F\left(q^{i}\right)}{q^{i}}\right)\left(\sum_{j=1}^{\infty} \frac{F\left(q^{j}\right)}{q^{j}}\right) .
\end{aligned}
$$

By (4.2) and (4.8), we see that $\int_{B(0 ; 1)} F\left(\left|A_{1}(\alpha)\right|\right) F\left(\left|A_{1}\left(T^{n} \alpha\right)\right|\right) \mathrm{d} \mu(\alpha)=P_{1}^{2}$. Therefore, by (4.7), we arrive at the hypothesis of Lemma 6 that $I=O(N)$, and this completes the proof of Theorem 1.

Proof of Theorem 2. The proof is similar to that of Theorem 1, so we shall give only an outline. First of all, we apply Lemma 6 with $S=B(0 ; 1)$,

$$
\begin{aligned}
\varphi(M, N ; \alpha)= & \mid \sum_{n=M+1}^{M+N}\left(H\left(\left|A_{1}\left(T^{n} \alpha\right)\right|, \ldots,\left|A_{m}\left(T^{n} \alpha\right)\right|\right)\right. \\
& \left.-\sum_{\left(i_{1}, \ldots, i_{m}\right) \in \mathbb{N}^{m}} H\left(q^{i_{1}}, \ldots, q^{i_{m}}\right)\left(\frac{(q-1)^{m}}{q^{i_{1}+\cdots+i_{m}}}\right)\right) \mid
\end{aligned}
$$

$\phi(N)=N$ and $p=2$. Next, by using the same idea of partition as in (4.1), we can calculate

$$
\int_{B(0 ; 1)} H\left(\left|A_{1}(\alpha)\right|, \ldots,\left|A_{m}(\alpha)\right|\right) \mathrm{d} \mu(\alpha)=\sum_{\left(i_{1}, \ldots, i_{m}\right) \in \mathbb{N}^{m}} H\left(q^{i_{1}}, \ldots, q^{i_{m}}\right)\left(\frac{(q-1)^{m}}{q^{i_{1}+\cdots+i_{m}}}\right) .
$$

Finally, if we put $P=\int_{B(0 ; 1)} H\left(\left|A_{1}(\alpha)\right|, \ldots,\left|A_{m}(\alpha)\right|\right) \mathrm{d} \mu(\alpha)$, then

$$
\int_{B(0 ; 1)} H\left(\left|A_{1}(\alpha)\right|, \ldots,\left|A_{m}(\alpha)\right|\right) H\left(\left|A_{1}\left(T^{n} \alpha\right)\right|, \ldots,\left|A_{m}\left(T^{n} \alpha\right)\right|\right) \mathrm{d} \mu(\alpha)=P^{2}
$$

These observations lead to Theorem 2 .

To prove Theorems $3-5$, we recall the following two elementary identities $\sum_{n=1}^{\infty} n x^{n}=\frac{x}{(1-x)^{2}}$ and $\sum_{n=1}^{\infty} n^{2} x^{n}=\frac{x(1+x)}{(1-x)^{3}}$ for $|x|<1$. We focus on the case when $x=q^{-1}$. 
Proof of Theorem 3. In view of Theorem 1, consider $F(x)=\log _{q} x$. By (4.2) and (4.3), we have

$$
\begin{gathered}
\int_{B(0 ; 1)} \log _{q}\left(\left|A_{1}(\alpha)\right|\right) \mathrm{d} \mu(\alpha)=(q-1) \sum_{n=1}^{\infty} \frac{n}{q^{n}}=\frac{q}{q-1} \\
\int_{B(0 ; 1)}\left(\log _{q}\left(\left|A_{1}(\alpha)\right|\right)\right)^{2} \mathrm{~d} \mu(\alpha)=(q-1) \sum_{n=1}^{\infty} \frac{n^{2}}{q^{n}}=\frac{q(q+1)}{(q-1)^{2}} .
\end{gathered}
$$

This completes the proof of Theorem 3 .

Proof of Theorem 4. Apply Theorem 1 with $F(x)=\frac{1}{(q-1)|A|} \chi_{\{|A|\}}(x)$, where $\chi_{E}(x)$ is the characteristic function of a set $E$. The reason that we use this function is to observe that $F\left(\left|A_{1}(\alpha)\right|\right)=\chi_{\{A\}}\left(A_{1}(\alpha)\right)$ for almost everywhere $\alpha \in B(0 ; 1)$ with respect to Haar measure. In other words, we have

$$
\frac{1}{N} \sum_{n=1}^{N} \chi_{\{A\}}\left(A_{1}\left(T^{n-1} \alpha\right)\right)=\frac{1}{N} \sum_{n=1}^{N} \frac{1}{(q-1)|A|} \chi_{\{|A|\}}\left(\left|A_{1}\left(T^{n-1} \alpha\right)\right|\right)
$$

for almost everywhere $\alpha \in B(0 ; 1)$ with respect to Haar measure.

By (4.2) and (4.3), we have

$$
\begin{gathered}
\int_{B(0 ; 1)} \frac{1}{(q-1)|A|} \chi_{\{|A|\}}\left(\left|A_{1}(\alpha)\right|\right) \mathrm{d} \mu(\alpha)=\left(\frac{q-1}{|A|}\right)\left(\frac{1}{(q-1)|A|}\right)=|A|^{-2} \\
\int_{B(0 ; 1)}\left(\frac{1}{(q-1)|A|} \chi_{\{|A|\}}\left(\left|A_{1}(\alpha)\right|\right)\right)^{2} \mathrm{~d} \mu(\alpha)=\left(\frac{q-1}{|A|}\right)\left(\frac{1}{(q-1)|A|}\right)^{2}=\frac{|A|^{-3}}{(q-1)} .
\end{gathered}
$$

This completes the proof of Theorem 4 .

Proof of Theorem 5. In view of Theorem 1, we consider $F_{1}(x)=\chi_{\left\{q^{l}\right\}}(x), F_{2}(x)=$ $\chi_{\left[q^{l}, \infty\right)}(x)$ and $F_{3}(x)=\chi_{\left[q^{k}, q^{l}\right)}(x)$, respectively. By (4.2) and (4.3), we have

$$
\begin{aligned}
\int_{B(0 ; 1)} \chi_{\left\{q^{l}\right\}}\left(\left|A_{1}(\alpha)\right|\right) \mathrm{d} \mu(\alpha) & =\frac{q-1}{q^{l}} ; \\
\int_{B(0 ; 1)}\left(\chi_{\left\{q^{l}\right\}}\left(\left|A_{1}(\alpha)\right|\right)\right)^{2} \mathrm{~d} \mu(\alpha) & =\frac{q-1}{q^{l}} ; \\
\int_{B(0 ; 1)} \chi_{\left[q^{l}, \infty\right)}\left(\left|A_{1}(\alpha)\right|\right) \mathrm{d} \mu(\alpha) & =(q-1) \sum_{n=l}^{\infty} \frac{1}{q^{n}}=\frac{1}{q^{l-1}} \\
\int_{B(0 ; 1)}\left(\chi_{\left[q^{l}, \infty\right)}\left(\left|A_{1}(\alpha)\right|\right)\right)^{2} \mathrm{~d} \mu(\alpha) & =(q-1) \sum_{n=l}^{\infty} \frac{1}{q^{n}}=\frac{1}{q^{l-1}}
\end{aligned}
$$




$$
\begin{gathered}
\int_{B(0 ; 1)} \chi_{\left[q^{k}, q^{l}\right)}\left(\left|A_{1}(\alpha)\right|\right) \mathrm{d} \mu(\alpha)=(q-1) \sum_{n=k}^{l-1} \frac{1}{q^{n}}=\frac{1}{q^{k-1}}\left(1-\frac{1}{q^{l-k}}\right) \\
\int_{B(0 ; 1)}\left(\chi_{\left[q^{k}, q^{l}\right)}\left(\left|A_{1}(\alpha)\right|\right)\right)^{2} \mathrm{~d} \mu(\alpha)=(q-1) \sum_{n=k}^{l-1} \frac{1}{q^{n}}=\frac{1}{q^{k-1}}\left(1-\frac{1}{q^{l-k}}\right) .
\end{gathered}
$$

This completes the proof of Theorem 5 .

\section{References}

1. V. Berthé AND H. NAKAdA, On continued fraction expansions in positive characteristic: equivalence relations and some metric properties, Expo. Math. 18(4) (2000), 257-284.

2. C. DE VROedt, Metrical problems concerning continued fractions, Compos. Math. 16 (1964), 191-195.

3. I. S. Gál and J. F. Koksma, Sur l'ordre de grandeur des fonctions sommables, Indag. Math. 12 (1950), 638-653.

4. V. HoundonougBo, Développement en fractions continues et répartititon modulo 1 dans un corps de séries formelles Thése de troisiéme cycle, Université de Bordeaux I, 1979.

5. M. IOSIFESCU AND C. KRAAIKAMP, Metrical theory of continued fractions, in Mathematics and its applications, Volume 547, pp. 225-232 (Kluwer Academic Publishers, Dordrecht, 2002).

6. A. YA. Khinchin, Continued fractions (Dover Publications, Mineola, NY, Russian edition, 1997). With a preface by B. V. Gnedenko, Reprint of the 1964 translation.

7. P. LeRTChoosakul and R. NAIR, On the metric theory of continued fractions in positive characteristic, Mathematika 60(2) (2014), 307-320.

8. H. NiEDERREITER, The probabilistic theory of linear complexity, in Advances in cryptology - Eurocrypt 88, Davos 88, Lecture Notes in Computer Science, Volume 330, pp. 195-197 (Springer, Berlin 1988).

9. W. M. Sснмidt, On continued fractions and Diophantine approximation in power series fields, Acta Arith. 95(2) (2000), 139-166.

10. V. G. SPRINDŽUK, Mahler's problem in metric number theory. Translated from the Russian by B. Volkmann. Translations of Mathematical Monographs, Volume 25, p. 99 (American Mathematical Society, Providence, RI, 1969). 